\title{
T2 and T2* Mapping
}

\author{
Goetz H. Welsch • Friedrich F. Hennig • \\ Sebastian Krinner $\cdot$ Siegfried Trattnig
}

Published online: 20 June 2014

(c) Springer Science+Business Media New York 2014

\begin{abstract}
New noninvasive biochemical quantitative magnetic resonance imaging (MRI) techniques are increasingly applied in cartilage repair and osteoarthritis (OA), where quantitative T2 mapping and initially also T2* mapping are seen as valid biomarkers. These quantitative MR biomarkers detect early degeneration of articular cartilage, mainly represented by increasing water content and a disruption of the collagen network. Recently, feasibility of biochemical MR imaging of repair tissue and the surrounding cartilage after cartilage repair procedures has been demonstrated. Different ultrastructural properties of the repair tissue after different repair procedures, such as microfracturing and autologous chondrocyte implantation, resulted in differences in imaging characteristics. Further, these biochemical imaging characteristics varied during follow-up and are able to depict repair tissue maturation. Within these approaches, zonal stratification analysis was considered crucial. Comparably in OA, T2 mapping seems to provide additional information during different stages of cartilage degeneration and can be used in patient subgroups as a predictor for disease progression. Although the results in various studies employing $\mathrm{T} 2$ and $\mathrm{T} 2 *$ mapping are very promising and valuable additional information on cartilage ultrastructure can be obtained, (comparable to all the other
\end{abstract}

This article is part of Topical Collection on Cartilage Imaging.

G. H. Welsch $(\bowtie)$ · F. F. Hennig · S. Krinner

Department of Trauma Surgery, University of Erlangen-

Nuremberg, Krankenhausstr. 12, 91054 Erlangen, Germany

e-mail: goetz.welsch@uk-erlangen.de;

welsch@bwh.harvard.edu

G. H. Welsch · S. Trattnig

Department of Radiology, MR Center, Medical University of

Vienna, Vienna, Austria quantitative cartilage imaging techniques) further multimodal investigation is needed especially since a standard of reference is hard to define.

Keywords MRI - Cartilage repair · Osteoarthritis · T2 mapping $\cdot$ T2* mapping

\section{Introduction}

These recent advances in MR sequences together with the implementation of high-resolution MRI due to high-field MR systems as well as sophisticated coil technology have overcome the existing limitations and led to promising in vivo approaches in morphological, especially in biochemical, MRI of cartilage, cartilage degradation as well as cartilage repair [1-3]. Significant advances have been made in characterizing, quantifying and standardizing the specific morphological as well as biochemical changes in patients with cartilage pathologies. Besides the exact evaluation of the cartilage defect and the cartilage degeneration, the specific therapeutical approaches can also be assessed in the best possible fashion noninvasively [4-6]. This precise assessment of the biochemical composition of articular cartilage was initially used in clinical approaches in the knee joint [7-9] and, some years later, also in the thinner articular cartilage of the hip joint $[10,11]$.

Concerning articular cartilage, standard MRI can visualise morphological alterations such as reduction in cartilage volume, cartilage contour irregularities, fissures and cartilage thinning [12]. As structural cartilage damage is preceded by biochemical alterations such as proteoglycan loss, or changes in the collagen matrix and the cartilage hydration, there is a substantial interest in detecting such changes in the course of cartilage disease/injury or after cartilage repair [13-15]. 


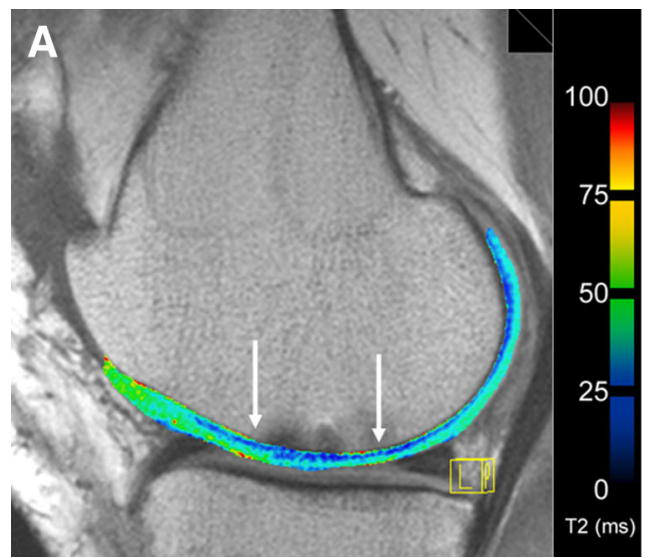

Fig. 1 a Sagittal multi-echo spin-echo (SE) T2 mapping sequence of the medial femoral condyle of a patient 24 months after microfracture therapy (MFX) with complete defect filling and integration of the cartilage transplant. The quantitative $\mathrm{T} 2$ values nevertheless show lower quantitative $\mathrm{T} 2$ values (marked by arrows) in the repair tissue compared to the surrounding cartilage. b Comparable sagittal SE T2

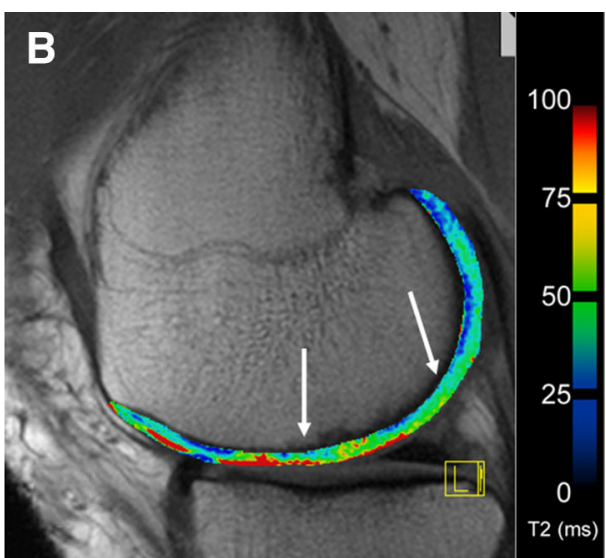

mapping sequence of the medial femoral condyle of a patient 24 months after matrix-associated autologous chondrocyte transplantation (MACT). The area of cartilage repair (arrows) shows comparable $\mathrm{T} 2$ relaxation times when comparing to the adjacent cartilage

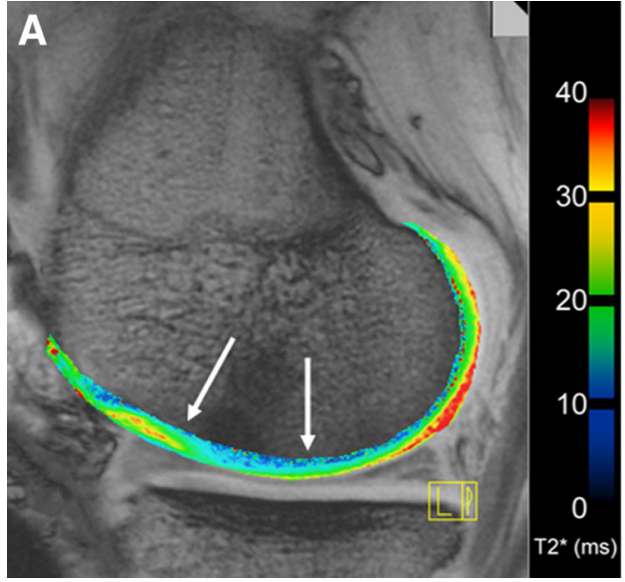

Fig. 2 a Sagittal gradient-echo (GRE) T2* mapping sequence of the medial femoral condyle of a patient 24 months after MFX. The quantitative $\mathrm{T} 2 *$ show reduced $\mathrm{T} 2 *$ values in the repair tissue (arrows) compared to the surrounding cartilage. b Comparable

When reporting on biochemical MR techniques, it is essential to know which tissue components are the images obtained by the respective techniques. Although when e.g. cartilage degradation starts, possibly every technique is influenced by a proteoglycan loss as well as by a change in the collagen matrix; these two main components (together with the tissue hydration) divide many of the available biochemical MR techniques. The biochemical MRI techniques most often reported to visualise cartilage ultrastructure are delayed Gadolinium-Enhanced MRI of Cartilage (dGEMRIC) and T2 mapping [16, 17]. dGEMRIC has the ability to quantify functionally relevant macromolecules within articular cartilage such as glycosaminoglycans (GAG). GAG are the main source of



sagittal gradient-echo (GRE) T2* mapping sequence of the medial femoral condyle of a patient 24 months after MACT. The area of cartilage repair (arrows) shows slightly reduced T2* relaxation times when comparing to the native surrounding cartilage

fixed charge density in cartilage, which are often decreased in the early stages of cartilage degeneration and are considered as a key factor in the progression of cartilage damage. On the other hand, T2 relaxation time mapping reflects the interaction of water and the extracellular matrix. Changes in hydration as well as collagen anisotropy, reported to be early indicators of cartilage deterioration, can be visualised by $\mathrm{T} 2$ relaxation time mapping. In cartilage repair, quantitative T2 mapping is able to assess the zonal structure of the repair tissue, and hence the maturation of the repair tissue over time [18]. Besides the standard spin-echo, multi-echo T2 mapping technique, T2* mapping could be a good alternative because it combines the benefits of biochemical cartilage evaluation with 
remarkable features including short imaging time and the ability of high-resolution three-dimensional imaging possibilities.

In scientific as well as clinical background, the role of $\mathrm{T} 2$ as well as $\mathrm{T} 2 *$ mapping is able to assess the collagen matrix ultrastructure and especially its zonal appearance. This zonal structure, visualised and quantified by increasing $\mathrm{T} 2$ or $\mathrm{T} 2 *$ relaxation times from deep to superficial cartilage, has been shown as a clear proof or hyaline (like) cartilage structure and might provide a more valuable measure compared to the bulk (mean) quantitative T2/T2* values [19, 20].

$\mathrm{T} 2$ as well as $\mathrm{T} 2 *$ mapping is comparably important in the follow-up after cartilage repair procedures, where hyaline-like repair tissue with a normal or nearly normal collagen matrix is seen to have positive predictive values [21]. In hip disorders like hip dysplasia or femuro-acetabular impingement (FAI), the collagen matrix as well as the tissue hydration is seen as a sensible marker in e.g. describing typical cartilage pathologies very early in their pathophysiological pathway [22, 23•]. In cartilage repair, there are different studies available which use T2 mapping techniques to e.g. i) differentiate in between the native cartilage and the repair tissue, ii) differentiate in between different cartilage repair tissues, iii) to assess the 'hyaline like' cartilage ultrastructure of the repair tissue, iv) to assess the maturation of the repair tissue over time and $v$ ) to assess the cartilage quality of the rest of the joint; what becomes more and more important as the 'health' of the whole joint is one very important key to the long-term success of cartilage repair surgeries.

\section{Basics in Imaging the Collagen Network by $\mathrm{T} 2$ or T2* Mapping}

As mentioned above, to visualise the biochemical content of articular cartilage different methodologies are available. Depending on the anatomical localization and the available (multichannel) coils, within the different large (knee, hip and ankle) and small (finger) joints as well as within the spine, compositional MR imaging can be achieved to assess the constitution of these tissues. T2 relaxation time mapping is the most widely used technique and was first to describe the composition of hyaline or hyaline-like articular cartilage in the knee joint, visualising its collagen matrix and tissue hydration. In initial studies, Goodwin et al. [24] found that the collagen fibre orientation, through $\mathrm{T} 2$ effects, dominantly influences the appearance of layers in hyaline cartilage in long-repetition-time MR images, where proton density is not a major factor, and the collagen fibre orientation in the transitional zone is not totally random. Furthermore, the same group reported in an in vitro study that the three-dimensional organization of collagen in cartilage has a strong influence on the MR appearance of cartilage in quantitative T2 mapping. This influence is caused by the restriction of water mobility and the resulting magic angle effect caused by curvature of the collagen network, possibly because of the influence on proteoglycan orientation [25]. The proteoglycan content itself, however, cannot be sufficiently imaged by quantitative T2 mapping and remains subject to other biochemical methodologies.

The group of Dardzinski et al. studied intensively the resulting spatial variation of $\mathrm{T} 2$ in human articular cartilage and found that there is a reproducible pattern of increasing $\mathrm{T} 2$ that is proportional to the known spatial variation in cartilage water and is inversely proportional to the distribution of proteoglycans. The authors postulate that these regional $\mathrm{T} 2$ differences are secondary to the restricted mobility of cartilage water within an anisotropic solid matrix [26]. Shortly afterwards, this group proofed this spatial pattern of $\mathrm{T} 2$ relaxation time values in vivo and showed a consistent pattern of spatial variation in cartilage T2 with longer values near the articular surface. The greatest variation occurs in the patella, where T2 increases from $45.3 \mathrm{~ms}$ (milliseconds) at a normalized distance of 0.33 and $67.0 \mathrm{~ms}$ at a distance of 1.0. These are the first results to demonstrate the feasibility of in vivo T2 mapping of the femoral tibial cartilage. The spatial variation of the femoral and the tibial cartilage seem to be roughly comparable to patellar cartilage; however, the increase in T2 values seem to be little less pronounced [9].

Mosher et al. were able to obtain different studies on the use of quantitative T2 mapping in vivo mainly in volunteer studies [27-31]. Among other findings, they were able to characterize the effects of age and physical activity level on cartilage thickness and especially T2 response immediately after running. Running results in a measurable decrease in cartilage thickness and MRI T2 values of superficial cartilage, consistent with greater compressibility of the superficial cartilage layer [29]. Furthermore, the effect of gender on in vivo cartilage magnetic resonance imaging T2 mapping was studied-where in young, healthy volunteers, the magnitude and the spatial dependency of cartilage T2 do not differ with gender [27].

In terms of osteoarthritis (OA), in a very recent review article, Mosher et al. [32] state that imaging plays a major role in $\mathrm{OA}$ research with publications heavily focused on quantitative and semiquantitative MRI evaluation of the knee with increasing application of compositional MRI techniques in the hip. Translational and clinical research application of compositional MRI techniques and especially T2 mapping is becoming more common driven in part by the data from the Osteoarthritis Initiative (OAI). In a study employing quantitative T2 mapping from the OAI, Joseph et al. [33•] report that the prevalence of knee 
abnormalities significantly increased over 3 years in the OAI cohort with increased cartilage $\mathrm{T} 2$ at baseline, which predicts the longitudinal morphological degeneration in the cartilage, meniscus and bone marrow over 3 years in subjects with risk factors for OA. This represents a very important finding as $\mathrm{T} 2$ relaxation times are used to provide a predictor or 'biomarker' for following the changes. Another attempt to use quantitative T2 as a 'biomarker' in OA was presented by Jungmann and co-workers [34•.] who found metabolic risk factors to be associated with higher $\mathrm{T} 2$, suggesting that increased cartilage degeneration may be caused by modifiable metabolic disorders. Another study based on data from the OAI reports on middle-aged asymptomatic individuals with risk factors for knee OA. These were seen to have high prevalence of cartilage and meniscus knee lesions, where physically active individuals had more knee abnormalities and higher patellar T2 values [35]. These studies provide evidence for the use of $\mathrm{T} 2$ mapping in the onset of $\mathrm{OA}$, where $\mathrm{T} 2$ relaxation times may be used as a predictive 'biomarker'.

As mentioned above, besides the knee joint, also the cartilage of the hip joint and initially of the ankle, the shoulder, the elbow and the wrist are the goal of biochemical T2 mapping studies. As large patient groups suffer from cartilage degeneration of different joints, describing articular cartilage at high fields is becoming increasingly important. Besides idiopathic OA, reasons for the development of $\mathrm{OA}$ are cartilage lesions in younger patients due to repetitive (micro-)trauma or overuse which may lead to the early onset of OA. Hence, surgical and conservative treatment options need sophisticated imaging to visualise their potential benefit. Basically, articular cartilage is a complex, dense, connective tissue that relies on the diffusion of solutes for its nutrition [36]. Responsible for the biomechanical properties of articular cartilage is the extracellular matrix, mainly composed of water ( $\sim 75 \%)$, collagen $(\sim 20 \%)$, and proteoglycan aggregates $(\sim 5 \%)$ [36, 37]. Water either freely moves throughout the matrix or is bound to macromolecules. Collagen in hyaline cartilage is largely type II, which provides a stable network and resists shear forces. The negatively charged proteoglycans are composed of a central core protein to which glycosaminoglycans (GAG) are bound. Articular cartilage is stratified primarily according to the orientation of collagen within a three-dimensional network [37, 38].

The most often implemented biochemical MR technique that assesses the collagen content of articular cartilage is $\mathrm{T} 2$ mapping [39]. In addition to quantitative T2 mapping of articular cartilage, recently, T2* relaxation time mapping is being discussed for the depiction of the collagen matrix [40]. For both methodologies, according to the stratification of cartilage from the subchondral bone to the cartilage surface, zonal evaluation and thus stratification of articular cartilage is crucial.

Besides articular cartilage, in the knee joint, especially the meniscus gets more and more in the focus of $\mathrm{T} 2 *$ relaxation time mapping. Normal human meniscus tissue has been found to be composed of $\sim 70 \%$ water, $\sim 25 \%$ collagen and $\sim 1 \%$ glycosaminoglycans with a regional variation due to the location in the knee joint. The predominantly dense framework is based on type I collagen fibres in a mainly circumferential orientation [41]. Within the meniscus, T2* mapping with short or ultra-short echo times is recently used with promising results.

One other large group of orthopedic patients, besides articular cartilage problems, are people suffering from (low) back pain. Degenerative disc disease is regarded as the most prevalent cause of low back pain, even though the pathophysiological correlations between pain and disc degeneration are not fully understood. In intervertebral discs (IVD), the annulus fibrosus (AF) consists of fibrocartilage and its function as a rigid containment for the nucleus pulposus (NP) accounts for its fibrous structure and low water content. The gelatinous structure of the NP, however, consists mostly of water, bearing a low yield of collagenous material. This combination of an intact $\mathrm{AF}$ together with a, thus, working NP is the key to movement and suspension in the biomechanical framework of the spine. Degeneration of IVDs is initiated by the incapacity of disc cells to maintain a highly hydrated proteoglycanrich matrix of the NP as well as a loss of the collagen structure that affects the mechanical integrity of the IVD. Due to the high reliance of the IVDs on their biochemical composition and thus its biomechanical function, compositional MR techniques are getting increasingly important in the diagnosis and treatment follow-up of patients with LBP. Especially T2 and also T2* mapping are able to visualise the IVDs. Comparable to the articular cartilage, also for the assessment of the IVDs, the biochemical stratification from the AF to the NP needs to be visualised.

\section{T2 Relaxation Time Mapping of Articular Cartilage}

The transverse relaxation time (T2) of cartilage is a sensitive parameter for the evaluation of changes in water and collagen content and tissue anisotropy [39]. Quantitative $\mathrm{T} 2$ values (ms) of cartilage reflect the interaction of water and the extracellular matrix on a molecular level. The collagen fibre orientation defines the layers of articular cartilage which is of outmost importance when imaging articular cartilage and cartilage repair tissue or degenerated cartilage. Thus, the three-dimensional organization of the collagen network, influenced by water mobility, the 
proteoglycan orientation and the resulting magic angle at $55^{\circ}$ (with respect to the main magnetic field (B0), where the magic angle is a physical phenomenon influencing image characteristics), influence the appearance of quantitative $\mathrm{T} 2$ values $[38,42]$. In healthy articular cartilage, an increase in $\mathrm{T} 2$ values from deep to superficial cartilage layers can be observed, based on the anisotropy of collagen fibres running perpendicular to cortical bone in the deep layer of cartilage [43]. Histologically validated animal studies have shown this zonal increase of the quantitative $\mathrm{T} 2$ values as a marker of hyaline or hyaline-like cartilage organization after cartilage repair procedures in the knee $[44,45]$. To visualise this zonal variation in vivo, high spatial resolution is essential, which can be achieved at high-field MR (1.5-3.0 Tesla), together with dedicated multi-channel coils in clinical approaches [46-48].

These studies have demonstrated its feasibility as well as the benefit of a functional evaluation of articular cartilage to see pre-morphological abnormalities. Studies comparing T2 relaxation at $1.5 \mathrm{~T}$ and $3.0 \mathrm{~T}$ reported a less pronounced dependence on the magnetic field strength compared to longitudinal T1 relaxation; however, a shortening of T2 values at $3.0 \mathrm{~T}$ by approximately $10 \%$ and by $10-20 \%$ at 4.0T compared to $1.5 \mathrm{~T}$ have been published [49-51]. Nevertheless, quantitative $\mathrm{T} 2$ values reported in literature are to a great extent depending on the applied sequence. Stanisz et al. [50] found their $\mathrm{T} 2$ values at both 1.5T and 3.0 T to be similar to those of Smith et al. [43] but higher compared to the evaluation of Gold et al. [49]. All three study groups, however, used different sequences with in part different sequence parameters in their quantitative evaluation of T2. Our own group uses a traditional multi-echo spin-echo technique for standard T2 mapping; nevertheless, the comparison between these techniques remains challenging and we strongly recommend the studies to keep the sequence, the coil, the employed scanner and thus the complete study protocol the same. Otherwise, longitudinal and cross-sectional comparisons might be not valid.

\section{T2 Mapping in Cartilage Repair}

In cartilage repair tissue, nevertheless, global (bulk; full thickness) $\mathrm{T} 2$ and especially zonal $\mathrm{T} 2$ evaluations have shown very promising results. Hence, in the follow-up after matrix-associated autologous chondrocyte transplantation (MACT), the visualisation of cartilage repair tissue maturation was possible and a differentiation between cartilage repair tissue at the patella and the medial femoral condyle could be achieved [48, 52, 53]. Furthermore using T2 mapping, cartilage repair tissues after different repair procedures could be distinguished [54]; whereas cartilage repair tissue after MFX-histologically seen as fibrocartilage-shows no zonal increase from deep to superficial cartilage aspects and repair tissue after MACT —-histologically reported as hyaline-like—shows a significant stratification.

The results in cartilage repair in terms of maturation of the repair tissue were shown to be dependent on the underlying cartilage repair procedure as well as on the different follow-up intervals which were reported during the maturation of the repair tissue. Here it has to be taken into consideration that, over time, the repair tissue as well the whole joint might also show degenerative changes (which are again based on the age of the patient and the pre-operative status of the joint as well as on possible parallel surgical procedures like ACL or meniscus surgery or high tibial osteotomy).

Holtzman et al. reported that 3-6 months and 1 year after OCT, T2 values showed no significant difference between repair cartilage and normal cartilage [55]. Salzmann et al. found that $\mathrm{T} 2$ values were higher in repair tissue after OCT compared to normal cartilage and MACI repair tissue 3.5 years after surgery [56]. Krusche-Mandl et al. also found a significant difference of T2 values between repair cartilage after OCT and normal cartilage 8 years after surgery [57•].

Maturation processes were also described for MFX. Oneto et al. [58] published a case study with an arthroscopic correlation. They found initially elevated $\mathrm{T} 2$ values for MFX repair tissue, which approached values of normal cartilage after two years in the case of graft integrity and lower T2 values for therapy failure. In another study, Theologis et al. examined patients after MFX and found at 3-6 months after surgery that MFX repair tissue had significantly higher full-thickness $\mathrm{T} 2$ values relative to the normal cartilage [59]. At 1 year, T2 values of repair tissue decreased to values comparable to the normal cartilage. Repair tissue after bone marrow-derived cell transplantation at the talus was found to show comparable T2 values to hyaline cartilage at 2-year and 4-year follow-ups [60, 61]. In two different studies, about 2.3 and 2.7 years after surgery, our group found reduced $\mathrm{T} 2$ in cartilage repair areas after MFX; whereas after MACI, T2 was similar to the normal cartilage [15, 62].

This leads to the very important zonal analyses of the repair tissue especially when assessing the maturation (and possibly also the degradation over time) of the repair tissue (and the rest of the joint). T2 of the repair tissue after MFX showed zonal stratification at 3-6 months, which was lost after 12 months [59]. This initial zonal variation, nevertheless, can also be due to the increased hydration of the repair tissue which might be more pronounced in the superficial cartilage areas shortly after surgery. Most studies, however, reported that normal zonal variation was lacking after MFX [15, 63-66]. 
Tissue maturation processes can also be depicted after ACI [63]. Kurkijarvi et al. showed that T2 values for ACI repair tissue were higher and more heterogeneous than T2 of the normal control cartilage about one year after surgery with a lack of zonal organization. Our group found higher $\mathrm{T} 2$ relaxation times for repair tissue than for normal cartilage 3-13 months after ACI, but found no difference after 19-42 months [67]. In another study, we were able to show that there was no significant difference in T2 values of repair cartilage compared to normal cartilage 20 months after MACI [18]. Correspondingly, Eshed et al. [68] reported that $\mathrm{T} 2$ values were lower in patients with MACI with longer duration implants (6-49 months post surgery). According to Salzmann et al. [56], repair tissue had significantly lower $\mathrm{T} 2$ values than the normal cartilage about 3.5 years after surgery MACI. At the talus, ACI repair tissue showed normal T2 values in 5-year and 10-year follow-ups [69, 70].

The zonal differences between the cartilage layers have been reported previously [15, 63, 71, 72]. However, these zonal differences seem to vary during the maturation process after ACI. After one year, no zonal stratification was detected [73]. At later time points, repair tissue after MACI showed a significant T2 increase from deep to superficial zones comparable to the normal cartilage $[15,65]$. Within the maturation process, also the zonal stratification as measures by $\mathrm{T} 2$ relaxation times seems to approach the values of control cartilage during the second year after MACI [67]. In another study, zonal stratification did not exist in repair tissue 20 months after surgery, but during a year of follow-up repair tissue matured and a zonal stratification appeared, while morphological evaluation showed no significant difference between the baseline and the 1-year follow-up MRI [18]. About 28 months after surgery, for ACI an increase of T2 from deep to superficial zone was found as opposed to no zonal variation for MFX [15]. Also 5.5 years after surgery, zonal stratification in MACI repair tissue was detected [74•]. Overall, these findings for $\mathrm{T} 2$ relaxation time measurements of cartilage repair tissue may correspond to the described histological differences of the tissue and indicate a maturation of both layers with a decrease in water content and an increase of collagen content and orientation [62].

In a biomechanical approach our group analysed biomechanical characteristics of repair tissue after MACI [75••]. In extended knees, there was no significant difference between T2 in volunteers and patients; however, during 45 degrees flexion (compression), T2 values within volunteers were significantly increased in contrast to $\mathrm{T} 2$ values in patients, most likely due to a lack of biomechanical adjustment of repair tissue [75••]. Another study pronounced differences between T2 values acquired at the end of the MR examination (compared to the beginning of the MR examination) for MACI repair tissue compared to control cartilage
[76]. Zonal T2 measurements revealed a higher dependency on unloading for the superficial cartilage layer [76]. This 'unloading' approach with a T2 mapping sequence directly after the beginning of the MRI (early unloading) and a T2 mapping sequence at the end of the MR protocol (late unloading) may provide the easiest way of biomechanical MRI. The patient has to load the knee prior to MRI e.g. by walking up and down the stairs, and unload the knee during MRI. This approach showed not only promising results in MACT patients, but also degenerative cartilage changes $[77 \bullet, 78 \bullet \bullet$. Hence, the change in quantitative T2 values during unloading might be a very sensitive parameter to assess the subtle changes in cartilage ultrastructure.

Regarding different knee compartments, the MOCART score showed no significant differences between MACI at the patella and MFC [53], but higher T2 values were found at the MFC repair tissue [53]. The zonal increase in T2 values from deep to superficial had an earlier onset in the repair tissue of the patella compared to the MFC after MACI [53]. Further analyses compared different MACI scaffolds. Higher T2 values were described for collagenbased scaffold compared to hyaluronic-based scaffold for MACI 2 years after surgery [79]. Figure 1 represents a clinical example of T2 mapping after cartilage repair.

Studies reported that $\mathrm{T} 2 *$ may provide additional information on cartilage repair tissue [63]. T2* has faster imaging times and the possibility of 3D acquisition, thereby providing greater spatial resolution [66]. Stelzeneder et al. [80] reported that at 1 year follow-up after arthroscopic autologous collagen-induced chondrogenesis (ACIC), T2* was not significantly different between the repair tissue and normal cartilage, using a GRE T2* approach. By employing the same T2* sequence, we found 2.3 years after MACI a comparable T2 between the repair tissue and normal cartilage, but lower T2* in repair tissue. Zonal stratification was also more pronounced for $\mathrm{T} 2 *$ than for $\mathrm{T} 2$ [81]. Considering the correlation between the $\mathrm{T} 2$ and the $\mathrm{T} 2 *$ measurements, a clearly significant association between the two relaxation times is notable, however, with relatively low correlation coefficients especially for the deep cartilage layer. This might be due to the influence of local susceptibility fields on $\mathrm{T} 2 *$ which can operate at a macroscopic level, i.e. at the bone/cartilage interface, or at the microscopic level, i.e. associated with the underlying microstructure of the cartilage. This also accounts for the differences in the zonal stratification between $\mathrm{T} 2$ and $\mathrm{T} 2 *$, with a clearer increase from deep to superficial relaxation times in $\mathrm{T} 2 *$ than in T2. For MFX, comparable to MSME T2 mapping, no spatial variation was observed for cartilage repair tissue using T2* mapping [66]. At 7T, T2 and T2* mapping showed to be applicable, however, with overall lower relaxation times and a reduced zonal appearance of articular cartilage [82]. Figure 2 represents a clinical example of $\mathrm{T} 2 *$ mapping after cartilage repair. 


\section{Clinical Potential and Conclusion}

The clinical potential of $\mathrm{T} 2$ and $\mathrm{T} 2 *$ mapping is getting obvious when looking at the presented studies. Hence, the additional information on the cartilage quality can answer clinical questions; especially, since it is a valid parameter for cartilage repair tissue maturation as well as differentiation after various repair techniques. Thus, $\mathrm{T} 2$ mapping has to be seen as a valuable tool in advanced diagnostics. As mentioned above, this benefit is present in the diagnosis of cartilage irregularities and in the follow-up after different treatment procedures. Surgical and, in future, more and more nonsurgical regenerative cartilage approaches can be assessed and a quantification of the results and the biochemical cartilage structure is possible. Additionally, this cannot only be used for cartilage injuries or irregularities but also as a measure for cartilage and following joint health. Comparable to cartilage injury and cartilage repair, $\mathrm{T} 2$ relaxation time mapping is also used in various studies on OA and especially the results of the OA initiative are showing that quantitative $\mathrm{T} 2$ can be used as a biomarker and predict the ongoing of $\mathrm{OA}$. More predictive studies have to be carried in cartilage repair, where more studies are available; however, the populations are (mainly due to the various repair techniques and the lower number of comparable patients) much smaller.

Acknowledgments The work was supported by the Emerging Fields Initiative (EFI) and the EFI-Moves Project of the University of Erlangen-Nuremberg, Germany.

\section{Compliance with Ethical Guidelines}

Conflict of Interest Dr. Goetz H. Welsch, Dr. Friedrich F. Hennig and Dr. Sebastian Krinner each declare no potential conflicts of interest. Siegfried Trattnig is a section editor for Current Radiology Reports.

Human and Animal Rights and Informed Consent This article does not contain any studies with human or animal subjects performed by any of the authors.

\section{References}

Papers of particular interest, published recently, have been highlighted as:

- Of importance;

-. Of major importance

1. Potter HG, Black BR, le Chong R. New techniques in articular cartilage imaging. Clin Sports Med. 2009;28:77-94.

2. Welsch GH, Trattnig S, Domayer S, Marlovits S, White LM, Mamisch TC. Multimodal approach in the use of clinical scoring, morphological MRI and biochemical T2-mapping and diffusionweighted imaging in their ability to assess differences between cartilage repair tissue after microfracture therapy and matrixassociated autologous chondrocyte transplantation: a pilot study. Osteoarthr cartil. 2009.
3. Welsch GH, Zak L, Mamisch TC, Resinger C, Marlovits S, Trattnig S. Three-dimensional magnetic resonance observation of cartilage repair tissue (MOCART) score assessed with an isotropic three-dimensional true fast imaging with steady-state precession sequence at 3.0 Tesla. Invest Radiol. 2009;44:603-12.

4. Potter HG, le Chong R, Sneag DB. Magnetic resonance imaging of cartilage repair. Sports Med Arthrosc. 2008;16:236-45.

5. Trattnig S, Millington SA, Szomolanyi P, Marlovits S. MR imaging of osteochondral grafts and autologous chondrocyte implantation. Eur Radiol. 2007;17:103-18.

6. Welsch GH, Mamisch TC, Hughes T, Domayer S, Marlovits S, Trattnig S. Advanced morphological and biochemical magnetic resonance imaging of cartilage repair procedures in the knee joint at 3 Tesla. Semin Musculoskelet Radiol. 2008;12:196-211.

7. Gillis A, Bashir A, McKeon B, Scheller A, Gray ML, Burstein D. Magnetic resonance imaging of relative glycosaminoglycan distribution in patients with autologous chondrocyte transplants. Invest Radiol. 2001;36:743-8.

8. Mosher TJ, Smith H, Dardzinski BJ, Schmithorst VJ, Smith MB. MR imaging and T2 mapping of femoral cartilage: in vivo determination of the magic angle effect. AJR Am J Roentgenol. 2001;177:665-9.

9. Smith HE, Mosher TJ, Dardzinski BJ, et al. Spatial variation in cartilage T2 of the knee. J Magn Reson Imaging. 2001;14:50-5.

10. Kim YJ, Jaramillo D, Millis MB, Gray ML, Burstein D. Assessment of early osteoarthritis in hip dysplasia with delayed gadolinium-enhanced magnetic resonance imaging of cartilage. J Bone Joint Surg Am. 2003;85A:1987-92.

11. Watanabe A, Boesch C, Siebenrock K, Obata T, Anderson SE. T2 mapping of hip articular cartilage in healthy volunteers at 3T: a study of topographic variation. J Magn Reson Imaging. 2007;26:165-71.

12. Recht M, Bobic V, Burstein D, et al. (2001) Magnetic resonance imaging of articular cartilage Clin Orthop Relat Res:S379-396.

13. Trattnig S, Marlovits S, Gebetsroither S, et al. Three-dimensional delayed gadolinium-enhanced MRI of cartilage (dGEMRIC) for in vivo evaluation of reparative cartilage after matrix-associated autologous chondrocyte transplantation at 3.0T: preliminary results. J Magn Reson Imaging. 2007;26:974-82.

14. Watanabe A, Boesch C, Anderson SE, Brehm W, Mainil Varlet P. Ability of dGEMRIC and T2 mapping to evaluate cartilage repair after microfracture: a goat study. Osteoarthr Cartil. 2009;17:1341-9.

15. Welsch GH, Mamisch TC, Domayer SE, et al. Cartilage T2 assessment at 3-T MR imaging: in vivo differentiation of normal hyaline cartilage from reparative tissue after two cartilage repair procedures-initial experience. Radiology. 2008;247:154-61.

16. Burstein D, Velyvis J, Scott KT, et al. Protocol issues for delayed Gd(DTPA)(2-)-enhanced MRI: (dGEMRIC) for clinical evaluation of articular cartilage. Magn Reson Med. 2001;45:36-41.

17. Mosher TJ, Dardzinski BJ. Cartilage MRI T2 relaxation time mapping: overview and applications. Semin Musculoskelet Radiol. 2004;8:355-68.

18. Welsch GH, Mamisch TC, Marlovits S, et al. Quantitative T2 mapping during follow-up after matrix-associated autologous chondrocyte transplantation (MACT): full-thickness and zonal evaluation to visualize the maturation of cartilage repair tissue. J Orthop Res. 2009;27:957-63.

19. Welsch GH, Apprich S, Zbyn S, et al. Biochemical (T2, T2* and magnetisation transfer ratio) MRI of knee cartilage: feasibility at ultra-high field (7T) compared with high field (3T) strength. Eur Radiol. 2011;21:1136-43.

20. Welsch GH, Trattnig S, Hughes T, et al. T2 and T2* mapping in patients after matrix-associated autologous chondrocyte transplantation: initial results on clinical use with 3.0-Tesla MRI. Eur Radiol. 2009;20:1515-23. 
21. Knutsen G, Drogset JO, Engebretsen L, et al. A randomized trial comparing autologous chondrocyte implantation with microfracture. Findings at five years. J Bone Joint Surg Am. 2007;89:2105-12.

22. Bittersohl B, Hosalkar HS, Hughes T, et al. Feasibility of T2* mapping for the evaluation of hip joint cartilage at $1.5 \mathrm{~T}$ using a three-dimensional (3D), gradient-echo (GRE) sequence: a prospective study. Magn Reson Med. 2009;62:896-901.

23. - Bittersohl B, Miese FR, Hosalkar HS, et al. T2* mapping of acetabular and femoral hip joint cartilage at $3 \mathrm{~T}$ : a prospective controlled study. Invest Radiol. 2012;47:392-397. One recent study that provides for one of the first times the applicability of $T 2 *$ measurements in the thin hip cartilage. The possible benefits of $3 D$ gradient echo based $T 2 *$ mapping is discussed and its possible clinical use is shown.

24. Goodwin DW, Wadghiri YZ, Dunn JF. Micro-imaging of articular cartilage: $\mathrm{t} 2$, proton density, and the magic angle effect. Acad Radiol. 1998;5:790-8.

25. Goodwin DW, Zhu H, Dunn JF. In vitro MR imaging of hyaline cartilage: correlation with scanning electron microscopy. AJR Am J Roentgenol. 2000;174:405-9.

26. Dardzinski BJ, Mosher TJ, Li S, Van Slyke MA, Smith MB. Spatial variation of T2 in human articular cartilage. Radiology. 1997;205:546-50.

27. Mosher TJ, Collins CM, Smith HE, et al. Effect of gender on in vivo cartilage magnetic resonance imaging $\mathrm{T} 2$ mapping. J Magn Reson Imaging. 2004;19:323-8.

28. Mosher TJ, Dardzinski BJ, Smith MB. Human articular cartilage: influence of aging and early symptomatic degeneration on the spatial variation of T2-preliminary findings at $3 \mathrm{~T}$. Radiology. 2000;214:259-66.

29. Mosher TJ, Liu Y, Torok CM. Functional cartilage MRI T2 mapping: evaluating the effect of age and training on knee cartilage response to running. Osteoarthr Cartil. 2010;18:358-64.

30. Mosher TJ, Liu Y, Yang QX, et al. Age dependency of cartilage magnetic resonance imaging $\mathrm{T} 2$ relaxation times in asymptomatic women. Arthritis Rheum. 2004;50:2820-8.

31. Mosher TJ, Smith HE, Collins C, et al. Change in knee cartilage T2 at MR imaging after running: a feasibility study. Radiology. 2005;234:245-9.

32. Mosher TJ, Walker EA, Petscavage-Thomas J, Guermazi A. Osteoarthritis year 2013 in review: imaging. Osteoarthr Cartil. 2013;21:1425-35.

33. - Joseph GB, Baum T, Alizai H, et al. Baseline mean and heterogeneity of MR cartilage T2 are associated with morphologic degeneration of cartilage, meniscus, and bone marrow over 3 years-data from the Osteoarthritis Initiative. Osteoarthr Cartil. 2012;20:727-735. This study provided results of quantitative T2 mapping in a relatively big cohort of the Osteoarthritis Initiative (OAI). It is shown that quantitative T2 values are correlated to the morphological breakdown of articular cartilage.

34. • Jungmann PM, Kraus MS, Alizai H, et al. Association of metabolic risk factors with cartilage degradation assessed by $\mathrm{T} 2$ relaxation time at the knee: data from the osteoarthritis initiative. Arthritis Care Res (Hoboken). 2011;65:1942-1950. Another important study on quantitative T2 mapping in patients of the OAI. Very interestingly the T2 values can be correlated to other risk factors in the natural course of osteoarthritis.

35. Stehling $\mathrm{C}$, Liebl $\mathrm{H}$, Krug R, et al. Patellar cartilage: $\mathrm{t} 2$ values and morphologic abnormalities at 3.0-T MR imaging in relation to physical activity in asymptomatic subjects from the osteoarthritis initiative. Radiology. 2010;254:509-20.

36. Buckwalter JA, Mankin HJ. Articular cartilage: Degeneration and osteoarthritis, repair, regeneration, and transplantation. Instr Course Lect. 1998;47:487-504.
37. Poole AR, Kojima T, Yasuda T, Mwale F, Kobayashi M, Laverty $\mathrm{S}$. Composition and structure of articular cartilage-A template for tissue repair. Clin Orthop Relat Res. 2001;S26-S33.

38. Goodwin DW, Zhu HQ, Dunn JF. In vitro MR imaging of hyaline cartilage: correlation with scanning electron microscopy. Am J Roentgenol. 2000;174:405-9.

39. Mosher TJ, Dardzinski BJ. Cartilage MRI T2 relaxation time mapping: overview and applications. Semin Musculoskelet Radiol. 2004;8:355-68.

40. Welsch GH, Mamisch TC, Hughes T, et al. In vivo biochemical 7.0 Tesla magnetic resonance - Preliminary results of dGEMRIC, zonal $\mathrm{T} 2$, and $\mathrm{T} 2 *$ mapping of articular cartilage. Invest Radiol. 2008;43:619-26.

41. Messner K, Gao J. The menisci of the knee joint. Anatomical and functional characteristics, and a rationale for clinical treatment. J Anat. 1998;193(Pt 2):161-78.

42. Goodwin DW, Wadghiri YZ, Dunn JF. Micro-imaging of articular cartilage: $\mathrm{t} 2$, proton density, and the magic angle effect. Academic Radiology. 1998;5:790-8.

43. Smith HE, Mosher TJ, Dardzinski BJ, et al. Spatial variation in cartilage T2 of the knee. J Magn Reson Imaging. 2001;14:50-5.

44. Watrin-Pinzano A, Ruaud JP, Cheli Y, et al. Evaluation of cartilage repair tissue after biomaterial implantation in rat patella by using T2 mapping. Magn Reson Mater Phys Biol Med. 2004;17:219-28.

45. White LM, Sussman MS, Hurtig M, Probyn L, Tomlinson G, Kandel R. Cartilage T2 assessment: differentiation of normal hyaline cartilage and reparative tissue after arthroscopic cartilage repair in equine subjects. Radiology. 2006;241:407-14.

46. David-Vaudey E, Ghosh S, Ries M, Majumdar S. T-2 relaxation time measurements in osteoarthritis. Magn Reson Imaging. 2004;22:673-82.

47. Mosher TJ, Smith HE, Collins C, et al. Change in knee cartilage T2 at MR imaging after running: a feasibility study. Radiology. 2005;234:245-9.

48. Trattnig S, Mamisch TC, Welsch GH, et al. Quantitative T-2 mapping of matrix-associated autologous chondrocyte transplantation at 3 Tesla-An in vivo cross-sectional study. Invest Radiol. 2007;42:442-8.

49. Gold GE, Han E, Stainsby J, Wright G, Brittain J, Beaulieu C. Musculoskeletal MRI at 3.0 T: relaxation times and image contrast. AJR Am J Roentgenol. 2004;183:343-51.

50. Stanisz GJ, Odrobina EE, Pun J, et al. T1, T2 relaxation and magnetization transfer in tissue at 3T. Magn Reson Med. 2005;54:507-12.

51. Bolog N, Nanz D, Weishaupt D. Muskuloskeletal MR imaging at 3.0 T: current status and future perspectives. Eur Radiol. 2006;16:1298-307.

52. Welsch GH, Mamisch TC, Marlovits S, et al. Quantitative T2 mapping during follow-up after matrix-associated autologous chondrocyte transplantation (MACT): Full-thickness and zonal evaluation to visualize the maturation of cartilage repair tissue. J Orthop Res. 2009;27:957-63.

53. Welsch GH, Mamisch TC, Quirbach S, Zak L, Marlovits S, Trattnig S. Evaluation and comparison of cartilage repair tissue of the patella and medial femoral condyle by using morphological MRI and biochemical zonal T2 mapping. Eur Radiol. 2009;19:1253-62.

54. Welsch GH, Mamisch TC, Domayer SE, et al. Cartilage T2 assessment at 3-T MR imaging: in vivo differentiation of normal hyaline cartilage from reparative tissue after two cartilage repair procedures-initial experience. Radiology. 2008;247:154-61.

55. Holtzman DJ, Theologis AA, Carballido-Gamio J, Majumdar S, Li X, Benjamin C. T(1rho) and T(2) quantitative magnetic resonance imaging analysis of cartilage regeneration following 
microfracture and mosaicplasty cartilage resurfacing procedures. J Magn Reson Imaging. 2010;32:914-23.

56. Salzmann GM, Paul J, Bauer JS, et al. T2 assessment and clinical outcome following autologous matrix-assisted chondrocyte and osteochondral autograft transplantation. Osteoarthr Cartil. 2009; 17:1576-82.

57. - Krusche-Mandl I, Schmitt B, Zak L, et al. Long-term results 8 years after autologous osteochondral transplantation: $7 \mathrm{~T}$ gagCEST and sodium magnetic resonance imaging with morphological and clinical correlation. Osteoarthr Cartil. 2012;20:357-363. In a 7 Tesla approach very new and promising biochemical sequences (especially chemical exchange saturation transfer (CEST) are studied. Although these sequences are GAG specific (and not collagen specific like T2 mapping), the feasibility of CEST provides many possibilities in future studies.

58. Oneto JM, Ellermann J, LaPrade RF. Longitudinal evaluation of cartilage repair tissue after microfracture using T2-mapping: a case report with arthroscopic and MRI correlation. Knee Surg Sports Traumatol Arthrosc. 2010;18:1545-50.

59. Theologis AA, Schairer WW, Carballido-Gamio J, Majumdar S, $\mathrm{Li}$ X, Ma CB. Longitudinal analysis of T1rho and T2 quantitative MRI of knee cartilage laminar organization following microfracture surgery. Knee. 2012;19:652-7.

60. Battaglia M, Rimondi E, Monti C, et al. Validity of T2 mapping in characterization of the regeneration tissue by bone marrow derived cell transplantation in osteochondral lesions of the ankle. Eur J Radiol. 2011;80:e132-9.

61. Giannini S, Buda R, Battaglia M, et al. One-step repair in talar osteochondral lesions: 4-year clinical results and t2-mapping capability in outcome prediction. Am J Sports Med. 2013;41: 511-8.

62. Welsch GH, Trattnig S, Domayer S, Marlovits S, White LM, Mamisch TC. Multimodal approach in the use of clinical scoring, morphological MRI and biochemical T2-mapping and diffusionweighted imaging in their ability to assess differences between cartilage repair tissue after microfracture therapy and matrixassociated autologous chondrocyte transplantation: a pilot study. Osteoarthr Cartil. 2009;17:1219-27.

63. Nieminen MT, Nissi MJ, Mattila L, Kiviranta I. Evaluation of chondral repair using quantitative MRI. J Magn Reson Imaging. 2012;36:1287-99.

64. White LM, Sussman MS, Hurtig M, Probyn L, Tomlinson G, Kandel R. Cartilage T2 assessment: differentiation of normal hyaline cartilage and reparative tissue after arthroscopic cartilage repair in equine subjects. Radiology. 2006;241:407-14.

65. Welsch GH, Trattnig S, Scheffler K, et al. Magnetization transfer contrast and T2 mapping in the evaluation of cartilage repair tissue with 3T MRI. J Magn Reson Imaging. 2008;28:979-86.

66. Mamisch TC, Hughes T, Mosher TJ, et al. T2 star relaxation times for assessment of articular cartilage at $3 \mathrm{~T}$ : a feasibility study. Skeletal Radiol. 2012;41:287-92.

67. Trattnig S, Mamisch TC, Welsch GH, et al. Quantitative T2 mapping of matrix-associated autologous chondrocyte transplantation at 3 Tesla: an in vivo cross-sectional study. Invest Radiol. 2007;42:442-8.

68. Eshed I, Trattnig S, Sharon M, et al. Assessment of cartilage repair after chondrocyte transplantation with a fibrin-hyaluronan matrix-correlation of morphological MRI, biochemical T2 mapping and clinical outcome. Eur J Radiol. 2012;81:1216-23.

69. Battaglia M, Vannini F, Buda R, et al. Arthroscopic autologous chondrocyte implantation in osteochondral lesions of the talus: mid-term T2-mapping MRI evaluation. Knee Surg Sports Traumatol Arthrosc. 2011;19:1376-84.

70. Giannini S, Battaglia M, Buda R, Cavallo M, Ruffilli A, Vannini F. Surgical treatment of osteochondral lesions of the talus by open-field autologous chondrocyte implantation: a 10-year follow-up clinical and magnetic resonance imaging T2-mapping evaluation. Am J Sports Med. 2009;37(Suppl 1):112S-8S.

71. Trattnig S, Domayer S, Welsch GW, Mosher T, Eckstein F. MR imaging of cartilage and its repair in the knee-a review. Eur Radiol. 2009;19:1582-94.

72. Trattnig S, Mamisch TC, Pinker K, et al. Differentiating normal hyaline cartilage from post-surgical repair tissue using fast gradient echo imaging in delayed gadolinium-enhanced MRI (dGEMRIC) at 3 Tesla. Eur Radiol. 2008;18:1251-9.

73. Kurkijarvi JE, Mattila L, Ojala RO, et al. Evaluation of cartilage repair in the distal femur after autologous chondrocyte transplantation using T2 relaxation time and dGEMRIC. Osteoarthr Cartil. 2007;15:372-8.

74. - Domayer SE, Apprich S, Stelzeneder D, et al. Cartilage repair of the ankle: first results of T2 mapping at 7.0 $\mathrm{T}$ after microfracture and matrix associated autologous cartilage transplantation. Osteoarthr Cartil. 2012;20:829-836. This study provides important results of $T 2$ mapping in the ankle and the possibility to distinguish in between different cartilage repair procedures.

75. •- Pachowsky ML, Trattnig S, Wondrasch B, et al. In vivo evaluation of biomechanical properties in the patellofemoral joint after matrix-associated autologous chondrocyte transplantation by means of quantitative T2 MRI. Knee Surg Sports Traumatol Arthrosc. 2013. This study shows the sensitivity of T2 mapping in different positions (straight and bended knee) in relation to cartilage repair tissue and healthy cartilage. This study is very important as it combines quantitative T2 mapping with a biomechanical approach. It is shown that T2 mapping by reflecting the healthiness of cartilage is sensitive to the orientation of the collagen network in relationship to the main magnetic field and/ or loading.

76. Mamisch TC, Trattnig S, Quirbach S, Marlovits S, White LM, Welsch GH. Quantitative T2 mapping of knee cartilage: differentiation of healthy control cartilage and cartilage repair tissue in the knee with unloading-initial results. Radiology. 2010;254:818-26.

77. - Apprich S, Mamisch TC, Welsch GH, et al. (2011) Quantitative $\mathrm{T} 2$ mapping of the patella at 3.0T is sensitive to early cartilage degeneration, but also to loading of the knee. Eur J Radiol. 2011. This study shows the sensitivity of T2 mapping not only to cartilage degeneration but also to different stages of loading of the articular cartilage as a biomechanical evaluation.

78. •• Apprich S, Welsch GH, Mamisch TC, et al. Detection of degenerative cartilage disease: comparison of high-resolution morphological MR and quantitative T2 mapping at 3.0 Tesla. Osteoarthr Cartil. 2011;18:1211-1217. Another very important contribution on the correlation in between quantitative T2 mapping and cartilage degeneration. These studies are of outmost importance as they show the applicability of T2 mapping in the quantification of different stages of OA.

79. Welsch GH, Mamisch TC, Zak L, et al. Evaluation of cartilage repair tissue after matrix-associated autologous chondrocyte transplantation using a hyaluronic-based or a collagen-based scaffold with morphological MOCART scoring and biochemical T2 mapping: preliminary results. Am J Sports Med. 2010;38:934-42.

80. Stelzeneder D, Shetty AA, Kim SJ, et al. Repair tissue quality after arthroscopic autologous collagen-induced chondrogenesis (ACIC) assessed via T2* mapping. Skeletal Radiol. 2013;42:1657-64.

81. Welsch GH, Trattnig S, Hughes T, et al. T2 and T2* mapping in patients after matrix-associated autologous chondrocyte transplantation: initial results on clinical use with 3.0-Tesla MRI. Eur Radiol. 2010;20:1515-23.

82. Welsch GH, Mamisch TC, Hughes T, et al. In vivo biochemical 7.0 Tesla magnetic resonance: preliminary results of dGEMRIC, zonal T2, and T2* mapping of articular cartilage. Invest Radiol. 2008;43:619-26. 